\begin{tabular}{|l|l|}
\hline $\begin{array}{l}\text { Emigración internacional y políticas de vinculación : un estudio comparativo entre } \\
\text { Argentina y Uruguay }\end{array}$ & Titulo \\
\hline Modolo, Vanina - Autor/a; Vaccotti, Luciana - Autor/a; & Autor(es) \\
\hline En: Fomerco 2011. Río de Janeiro, Brasil. 14 al 16 de septiembre de 2011. & En: \\
\hline Buenos Aires & Lugar \\
\hline IIGG, UBA & Editorial/Editor \\
\hline 2011 & Fecha \\
\hline & Colección \\
\hline $\begin{array}{l}\text { Transnacionalismo; Relaciones internacionales; Políticas públicas; Emigración; } \\
\text { Argentina; Uruguay; }\end{array}$ & Temas \\
\hline Ponencias & Tipo de documento \\
\hline "http://biblioteca.clacso.edu.ar/Argentina/iigg-uba/20161125021326/modoloyvaccott.pdf" & URL \\
\hline $\begin{array}{l}\text { Reconocimiento-No Comercial-Compartir Igual CC BY-NC-SA } \\
\text { http://creativecommons.org/licenses/by-nc-nd/2.0/deed.es }\end{array}$ & Licencia \\
\hline
\end{tabular}

Segui buscando en la Red de Bibliotecas Virtuales de CLACSO http://biblioteca.clacso.edu.ar

Consejo Latinoamericano de Ciencias Sociales (CLACSO)

Conselho Latino-americano de Ciências Sociais (CLACSO)

Latin American Council of Social Sciences (CLACSO)

www.clacso.edu.ar

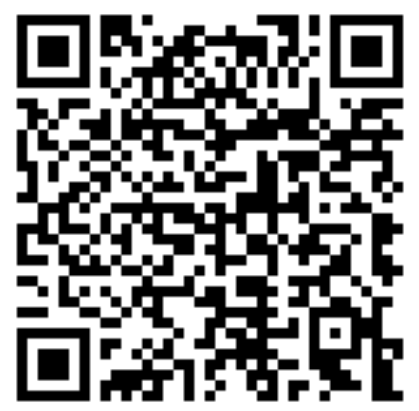




\section{Emigración internacional y políticas de vinculación: un estudio comparativo entre Argentina y Uruguay ${ }^{1}$}

Mg. Vanina Modolo, Doctoranda de la Facultad de Ciencias Sociales, Universidad de

Buenos Aires-Becaria CONICET, vaninamodolo@gmail.com

Lic. Luciana Vaccotti, Doctoranda de la Facultad de Ciencias Sociales, Universidad de

Buenos Aires-Becaria CONICET, lucianavaccotti@gmail.com

\section{Resumen}

Este trabajo se propone reflexionar sobre las políticas de vinculación con la emigración internacional a través del estudio comparativo de Argentina y Uruguay, en los que esta temática ocupa lugares muy distintos en la agenda política y, por ende, en las acciones implementadas en la materia. El trabajo se centra en los programas "Provincia 25" de Argentina y "Departamento 20" de Uruguay, con énfasis en las acciones implementadas por ambos países en el período reciente, y en el contexto de la problemática que representa la emigración internacional para ellos.

\section{Palabras Clave}

Emigración internacional-políticas públicas-vinculación-Argentina-Uruguay

\section{International Emigration and linking policies: a comparative analysis between Argentine and Uruguay}

\section{Summary}

This article presents a comparative study of policies towards international emigration in Argentina and Uruguay. In each of them international emigration deserves unequal relevance in the political agenda and, therefore, in the actions implemented in terms of linking emigrated populations to homeland. The study focuses on two instruments,

\footnotetext{
${ }^{1}$ Este trabajo se realiza en el marco del Proyecto UBACyT titulado "La cuestión migratoria en la Argentina: procesos de transformación, integración regional, derechos y prácticas sociales", Programación Científica
} 
"Province 25" in Argentina, and "Department 20" in Uruguay, with emphasis on actions taken by both countries in recent times, and in the context of the problem that international migration represents for them.

\section{Key Words}

International emigration-public policies-links-Argentina-Uruguay

\section{Emigración internacional y políticas de vinculación: un estudio comparativo entre Argentina y Uruguay}

\section{Transnacionalismo y vinculación}

El estudio de los vínculos entre los migrantes y sus países de origen adquiere un lugar en la agenda académica a partir de la década de 1990, cuando comienza una superación del enfoque dicotómico que consideraba a los migrantes simplemente como individuos que abandonaban un país y llegaban otro, y se incorpora la noción contemporánea de transnacionalismo aplicada a la migración. Así, la migración comienza a ser pensada como un proceso transnacional, que involucra relaciones multivinculadas (multi stranded) entre los migrantes y las sociedades de origen y destino (Vono 2006; Levitt y Nyberg-Sorensen 2004).

Desde esta perspectiva se considera que las prácticas transnacionales de los migrantes llevan a la formación de campos o espacios sociales transnacionales, entendidos como conjuntos de relaciones sociales entre los migrantes y sus diversas formas de organización en las sociedades de destino con los gobiernos y grupos en las sociedades de origen. Estas nociones resultan clave para el estudio de las políticas de vinculación de los Estados con sus poblaciones emigradas, en tanto superan la perspectiva nacionalista que considera a los Estados como único marco de la vida social, planteando así nuevas interrogantes (Vono 2006; Faist 2005; Levitt y Glick Schiller 2004; Guarnizo, Portes y Haller 2002; Glick Schiller et al 1999). 
La perspectiva transnacional constituye un marco de creciente aceptación para el estudio de las migraciones internacionales y sus efectos en las sociedades de origen y destino, en la medida en que proporciona una teoría para el desarrollo de tipologías y predicciones. Un importante número de investigaciones realizadas desde varias disciplinas académicas durante las últimas dos décadas años han analizado los vínculos sociales, políticos, económicos y culturales que establecen los migrantes con sus países de origen (Moraes 2009; Moraes, 2007; Escrivá 2009; Portes 2005; Guarnizo 2004; Guarnizo el at 2003; Pedone 2002; Glick Schiller et al 2001; Glick Schiller et al 1999, entre otras).

Aunque el tema de la vinculación de los emigrantes con su país de origen gana relevancia en la agenda de los países de la región desde comienzos del siglo XXI, las iniciativas orientadas tanto a conocer las formas en que los emigrantes se relacionan con el Estado y la sociedad de origen como a promover acercamientos entre éstos en relación a diversos temas, son más recientes (Vono 2006).

Además del rol de los migrantes como actores políticos, tanto en las sociedades de origen como de destino, las investigaciones que han abordado la dimensión política transnacional han enfatizado la importancia de las estrategias desarrolladas por los Estados de origen para vincularse con la población que reside fuera del país, promoviendo el lugar de la nación dentro de un campo social transnacional. En la actualidad existe una amplia literatura que busca contribuir a la reflexión en torno a las medidas adoptadas por los Estados—en tanto actores transnacionales-para reforzar sus vínculos con su población emigrada, así como sobre el rol de éstos en la reproducción de sujetos transnacionales (Guarnizo y Smith 1998). Algunos de los debates que tienen lugar en este campo-y que resultan especialmente relevantes para el tema de este artículo-tienen que ver con la posibilidad de considerar a las iniciativas de vinculación como "políticas", así como con los motivos de este creciente interés de los Estados por reforzar los vínculos con las comunidades de nacionales en el exterior (Moraes 2009).

Los impactos de la emigración en los países de origen han ocupado un lugar periférico en el debate en torno a las políticas migratorias, y los gobiernos de estos países, en general, han mantenido una actitud pasiva con respecto a los emigrantes y sus derechos. Con algunas excepciones puntuales, las gestiones orientadas a proteger los derechos de los emigrantes- 
en un marco de creciente presencia del tema de los derechos humanos de los migrantes en la agenda internacional—recién comienzan a ganar relevancia a partir de la última década del siglo $\mathrm{XX}$, con iniciativas bilaterales y multilaterales, entre ellas la adopción de la Convención Internacional sobre la Protección de todos los Trabajadores Migratorios y sus Familiares por parte de la Organización de las Naciones Unidas (1990). Paralelamente se asiste a una intensificación de la discusión sobre políticas de migración y desarrollo, que buscan que la migración se traduzca en beneficios económicos tanto para los países de origen como de destino. En este sentido, se han realizado numerosas reuniones internacionales orientadas a incluir temas migratorios en acuerdos de cooperación internacional. Asimismo, la Asamblea de la Organización de las Naciones Unidas de 2005 incorpora el tema de migración y desarrollo en su agenda, destacándose el tema de las remesas y la migración calificada (Cabella y Pellegrino 2007).

\section{Emigración internacional en Argentina y Uruguay}

Los efectos de las migraciones internacionales alcanzan actualmente a todo el planeta, diversificando los rasgos identificatorios de las sociedades posindustriales, en el marco de una tendencia global de concentración de movimientos migratorios entre países en vías de desarrollo y países desarrollados. La Organización de las Naciones Unidas estimó que el número de migrantes alcanzó a 191 millones en 2005, 115 millones de los cuales se dirigían hacia países desarrollados y 75\% de los cuales se concentraban en sólo 28 países (Cabella y Pellegrino 2007).

En este escenario, la discusión sobre políticas migratorias ha evolucionado hacia la "gobernabilidad de la migración", buscando superar la contradicción entre el proyecto de libre circulación de capital y de mercancías y el estricto control impuesto por las legislaciones nacionales a la instalación de trabajadores inmigrantes en sus territorios, para evitar conflictos surgidos de la competencia entre trabajadores nacionales e inmigrantes, el tráfico de personas y la sobre-explotación de los trabajadores migrantes en situación irregular, así como otros fenómenos como los nacionalismos y la xenofobia. La región no se encuentra ajena a esta discusión, que desafía visiones tradicionales en torno al "control" 
migratorio y la defensa de la soberanía, y evidencia las contradicciones y tensiones inherentes a la presente etapa de la globalización (Martínez Pizarro 2001).

\section{Argentina}

En Argentina, la emigración internacional aparece como una tendencia creciente a partir de la década de 1960 (Pellegrino 2003a). Las estimaciones disponibles señalan que hacia 1970 el volumen de argentinos residentes en el exterior no superaba los 150.000 , ascendiendo a 290.000 una década después (Gurrieri 1982). El stock de emigrantes correspondientes al período 1955-1984 oscila entre 499.000 y 609.000, lo que corresponde entre un 1,8\% y un 2,2\% de la población total del año 1980 (Bertoncello y Lattes 1986). Pellegrino sostiene que la emigración internacional constituye una tendencia estructural entre los sectores medios y medios altos con niveles educativos altos de la sociedad argentina (Pellegrino, 2003a).

Una estimación de la Organización Internacional para las Migraciones (OIM) de 2008 sostiene que el stock actual de emigrantes argentinos asciende a 806.369 personas, que representan un 2,1\% de la población total del país. Los principales países de destino de los argentinos son: España (229.009), Estados Unidos (144.023), Paraguay (61.649), Chile (59.637), Israel (43.718), Bolivia (36.261), Brasil (25.826), Uruguay (23.943), Canadá (14.877) e Italia (11.576) (OIM, 2008). Pellegrino también señala que la preocupación por la emigración internacional se debió a sus aspectos cualitativos, fundamentalmente asociados a la calificación de la población que abandonaba el país, que se caracterizó por elevados niveles educativos, así como por una fuerte presencia de profesionales y técnicos. La emigración internacional se masificó en la década de 1970_debido principalmente a la represión política y al aumento del desempleo-extendiéndose a un espectro más amplio de ocupaciones (Pellegrino 2003a).

\section{Uruguay}

La emigración internacional constituye una tendencia estructural de la población uruguaya en el siglo XX y la primera década del siglo XXI, como señalan los saldos migratorios negativos que se observan a partir de 1963. Se estima que durante el período 1963-1985, Uruguay tuvo un saldo migratorio negativo de 310.000 personas (12\% de la población media del período y $20 \%$ de la población económicamente activa). La emigración de este 
período se vio fundamentalmente motivada por el estancamiento económico y las limitaciones del modelo de desarrollo del país, así como por la inestabilidad política y social que culminó en la instalación de la dictadura en 1973 (Pellegrino, 2003b). Pellegrino (2009) afirma que, grosso modo, se puede estimar el volumen de personas nacidas en Uruguay que actualmente reside fuera del país en 600.000 , lo cual representa un $18 \%$ del total de la población.

Los principales destinos de la emigración de la década de 1970 fueron Argentina, Estados Unidos, Australia, Brasil, y España (Pellegrino, Vigorito y Macadar 2003), mientras que en los flujos más recientes se observa un aumento de la importancia Estados Unidos, España y otros destinos de larga distancia (Cabella y Pellegrino 2007; Pellegrino 2006). En el año 2000, las principales concentraciones de uruguayos se encontraban en Argentina, Estados Unidos, Brasil y España (87\% de la población nacida en Uruguay y censada en otros países) (Pellegrino, Vigorito y Macadar 2003).

El nivel educativo de los emigrantes del período es más elevado que el de la población que reside en Uruguay, aunque existen variaciones de acuerdo a los países de destino. La selectividad de los emigrantes aparece relacionada a la distancia de los países de destino así como a las características de sus mercados de trabajo (Cabella y Pellegrino 2007; Pellegrino 2006, 2003a)

\section{Políticas públicas de vinculación en Argentina y Uruguay Argentina}

En Argentina, la preocupación institucional por la población emigrada surge recién a mediados del siglo XX (Novick 2007). Las primeras acciones en materia de vinculación con la emigración datan de la década de 1960. Así, en 1965 se crea la Comisión Especial de Estudio de la Migración de Científicos, Profesionales, Técnicos y Obreros altamente calificados ${ }^{1}$. Años más tarde, en el Plan Trienal para la Reconstrucción y Liberación Nacional (1973-1977) aparece un capítulo dedicado a la formulación de una política referida a la emigración de argentinos concebida como un problema. 
En 1984, se crea la Comisión Nacional para el Retorno de los Argentinos en el Exterior, como órgano asesor del Poder Ejecutivo. En el marco de la misma, se difunde información sobre facilidades a retornantes: descuentos para transporte de efectos personales, reconocimiento y validez de estudios cursados en el exterior, títulos obtenidos en el exterior, amnistía a los desertores del servicio militar obligatorio e ingreso libre de gravámenes de efectos personas y del hogar, entre otras.

En 2003 surge el Programa R@ ICES (Red de Argentinos Investigadores y Científicos en el Exterior), dependiente del Ministerio de Educación de la Nación, cuyo propósito era el de impulsar el vínculo con investigadores argentinos residiendo en el exterior, promover su permanencia en el país y fomentar el retorno de aquellos interesados en desarrollar sus actividades en Argentina.

Calvelo (2007) sostiene que estas medidas se limitan al abordaje de perfiles emigratorios específicos (como la emigración altamente calificada), o a facilitar el retorno de la emigración forzada. No obstante, se observa una medida de carácter general: el otorgamiento del derecho al voto desde el exterior. La Ley $24.007^{2}$ de 1991 establece la condición de elector nacional para los ciudadanos argentinos residentes fuera del territorio nacional. Éstos deben estar inscriptos en el Registro de Electores Residentes en el Exterior ${ }^{3}$, y se tiene en cuenta el último domicilio acreditado en la Argentina para determinar el distrito al cual se le adjudicarán los votos emitidos. Estimaciones recientes de la Dirección Nacional de Migraciones ubican el volumen de argentinos que residen en el exterior en 1.053.000, cifra muy superior al padrón electoral de varias provincias argentinas. No obstante, la participación electoral de éstos ha sido escasa y decreciente ${ }^{4}$.

El decreto presidencial 254/2009 facilita la votación de los argentinos que residen en el exterior al permitir a los consulados habilitar mesas electorales. Según información de la Cancillería, el número de mesas electorales pasó de 114 en 2009 a 200 en 2011. El padrón electoral confeccionado de cara a las elecciones nacionales de octubre de 2011 incluye a 50.479 votantes inscriptos 5 .

Más allá de las medidas mencionadas, la normativa que le brinda un encuadre legal a la problemática de la emigración internacional es la Ley de Migraciones 25.871 de $2004^{6}$. Esta ley dedica tres artículos al tema emigratorio. El artículo $102^{7}$ insta a la firma de 
convenios con países de destino de la emigración argentina para garantizar mejores condiciones en materia de derechos laborales y seguridad social a los emigrantes. Asimismo, faculta al Poder Ejecutivo a tomar medidas de suspensión de beneficios contra los nacionales de países que impongan medidas que afecten el principio de reciprocidad.

Por su parte, el artículo $103^{8}$ facilita la introducción de efectos personales (vehículos, bienes muebles y bienes destinados a su actividad laboral) por parte de aquellos ciudadanos que desean retornar a sus respectivos países luego de dos años de residencia en el exterior. El Decreto Reglamentario aclara que la Dirección de Aduanas será la autoridad competente, y que quienes hayan gozado de este beneficio sólo podrán acogerse nuevamente a él después de transcurridos siete años, a partir de la fecha del acto administrativo por el que fue acordado.

Por último, el artículo $104^{9}$ dispone que las embajadas y consulados de la República Argentina deben contar con los servicios necesarios para informar a los argentinos en el exterior de las franquicias y demás exenciones para retornar al país.

\section{Provincia 25}

En 2007 se crea el programa Provincia $25^{10}$--en referencia a la organización administrativa y política del país en 24 provincias-bajo la competencia del Ministerio del Interior. El principal objetivo del programa es fortalecer los vínculos y la comunicación del Estado con los argentinos residentes en el exterior. Así, se propone garantizar el ejercicio de sus derechos políticos, promover su integración y la unificación de su representación, asistirlos en las gestiones públicas que sean competencia de este Ministerio y fortalecer los vínculos entre éstos y el Estado argentino.

Las principales líneas de acción están relacionadas con el ejercicio de los derechos electorales, la creación de institutos de representación de las comunidades de argentinos residentes en el exterior, promoviendo la integración de los mismos, la búsqueda de la representación parlamentaria específica de la población de Provincia 25, la facilitación de la gestión de documentación, el resguardo y la asistencia a los argentinos en el exterior en sus derechos sociales, el desarrollo y la implementación de herramientas de comunicación con la población de la Provincia 25, y la generación de la información necesaria sobre la 
población de Provincia 25, a fin de lograr una gestión eficaz de la política de vinculación ${ }^{11}$. Como sostiene Pazos (2009:133), este programa "constituye una forma novedosa de enfocar el problema y las posibles alternativas para la vinculación con los nacionales en el exterior, diferente de todas las precedentes, que aportaron conocimiento e información sobre el tema, pero no lograron su real participación".

A partir de la creación de este programa se ha llevado a cabo una serie de acciones. En mayo de 2008 se firma un Acta Acuerdo con IBM Argentina para el desarrollo del Programa "Volvé a Casa" para difundir las necesidades del mercado local en la industria informática entre los profesionales científicos argentinos comprendidos en la población objetivo del Programa Provincia 25, con el objetivo de integrarlos a la comunidad científica nacional. IBM se comprometió a aportar el material informativo del Programa "Volvé a Casa" y la Subsecretaría de Desarrollo y Fomento Provincial difundirá entre los científicos y tecnólogos argentinos radicados en el exterior a través del portal y la red del Programa Provincia 25 las posibilidades laborales y de investigación que IBM ponga a disposición.

En noviembre de 2008, a través del Programa Provincia 25 se firma un Convenio entre el Ministerio del Interior y el Banco de la Provincia de Buenos Aires por el cual el Banco dispone una atención especializada para los argentinos residentes en el exterior, considerando, entre otros servicios y beneficios, grupos de afinidad vinculados al envío de remesas desde el exterior, acceso a créditos en casos de repatriación, y una serie de productos y servicios financieros y no financieros adecuados a su situación específica y a las posibilidades que ofrece el mercado local. Entre sus objetivos específicos se encuentran el de implementar y consolidar sistemas de transferencias de fondos eficientes, seguros y en condiciones accesibles y propiciar la adecuación de otros productos y servicios que ayuden a facilitar la reinserción local y la de sus familias, poniendo los servicios bancarios formales a su alcance. A raíz de este Convenio se lanza "Provincia Ahorro 25" que consiste en una serie de instrumentos financieros tales como una Caja de Ahorros en pesos (cuenta habilitada para recibir transferencia desde el exterior, adherir débitos automáticos de impuestos y servicios de los bienes en el país, y acceso a las cuentas por intermedio del servicio de home banking, para consultas de saldos, movimientos y pago de impuestos y servicios), tarjeta Visa débito para los titulares de la cuenta y los adicionales que ellos 
designen, con uso tanto en cajeros automáticos de nuestro país y del exterior como en comercios adheridos a la marca Visa, caja de ahorro en dólares y el servicio de transferencias de fondos desde otros bancos corresponsales en el exterior ${ }^{12}$.

Además, se estableció un canal de consultas específicas con el Área Internacional de la Administración Nacional de la Seguridad Social (ANSES) y se gestionó la ampliación de acuerdos internacionales en la materia.

En diciembre de 2009 se llevó a cabo el segundo Encuentro de Argentinos en el Exterior bajo el lema "Por la Ampliación de la Nación", organizado por el programa Provincia 25. En la jornada se debatieron varios ejes, incluyendo las experiencias de argentinos en el exterior, el ejercicio del derecho al voto, el proyecto de ley de creación del distrito electoral exterior, la representación parlamentaria, la problemática de los científicos y profesionales en el exterior, la seguridad social, el envío de remesas, las propuestas de inversión, la Universidad Virtual de Quilmes y la Unidad Bicentenario, entre otros. Al encuentro asistieron ciudadanos argentinos residentes en 22 países, como Alemania, Brasil, Bolivia, Colombia, España, Estado Unidos, Francia, Guinea Ecuatoriana, Holanda, Islandia, Italia, Irlanda, Libia, México, Paraguay, Rep. Dominicana, Suecia y Suiza. El encuentro también contó con la participación de funcionarios públicos, docentes e investigadores.

Como surge de lo ya expuesto, uno de los temas principales que trabaja el programa es la asistencia a quienes desean retornar al país, para que la transición y las condiciones sean lo menos traumáticas posibles. Para ello, se elaboró una Guía para Argentinos que desean retornar, con una serie de consejos prácticos ${ }^{13}$.

En relación con uno de los objetivos prioritarios del Programa, se apoyó desde diversos sectores el proyecto de ley presentado por el diputado Ariel Pasini ${ }^{14}$ para la creación de un distrito exterior, que habilite una representación parlamentaria de cinco diputados por los argentinos residentes en el exterior. El proyecto busca la modificación del inciso 1 del artículo 39 del código electoral nacional (Ley 23.298).

\section{Uruguay}

Basándose en Supervielle (1989), Taks (2006) señala algunas de las principales características de las políticas migratorias del siglo XX en Uruguay, afirmando que las 
mismas: a) se orientan al control de procesos inmigratorios; b) se centran en tres objetivos: poblacional, económico y político; c) se basan en tres modelos: intentos de colonización agrícola, incentivo a artesanos urbanos especializados, e inmigración de personas altamente calificadas; y d) se orientan a la restricción de ingreso/expulsión de personas y grupos contra-hegemónicos y militantes (durante los períodos de autoritarismo, 1930-1935 y 19701984). Por su parte, Aguiar (en Taks 2006) sostiene que entre 1976 y 1985 las políticas públicas se caracterizaron por un escaso nivel de intervención y atención a los procesos migratorios.

Esta situación se modifica a partir de la década de 1980. En 1985 se crea la Comisión Nacional de Repatriación, orientada a coordinar los esfuerzos de organismos públicos y organizaciones no gubernamentales que buscaban la reinserción de los uruguayos que retornaban al país tras la dictadura. A nivel de la sociedad civil, las principales entidades que trabajaron con esta comisión oficial fueron la Comisión Nacional para el Reencuentro de los Orientales (1984) y el Servicio Ecuménico de Reinserción (1985). Notaro señala que la creación de esta comisión marca un cambio en la política migratoria del país, que pasa de promover la inmigración a priorizar el retorno de emigrantes. Ya en ese entonces, expertos consideraron que la emigración de uruguayos aumentaría por razones estructurales, abriendo dos posibles caminos: considerar la emigración de uruguayos como un fenómeno coyuntural o tomarla como una constante de los próximos veinte años; esto último señalaría la importancia de definir una política orientada a salvaguardar la identidad nacional y aprovechar los escasos recursos disponibles de la manera más adecuada posible (Notaro en Taks 2010, 2006). No obstante, estos cambios no fueron significativos, sino más bien intervenciones puntuales, visualizándose a la política económica como principal instrumento de retención. Esta visión persiste hasta comienzos del siglo XXI, confirmando la inexistencia de políticas migratorias que aborden el fenómeno de la emigración internacional, que es considerado "de naturaleza económica” (Supervielle en Taks 2006).

Este panorama comienza a transformarse en 2001, con la puesta en marcha del Programa de Vinculación con Uruguayos Altamente Calificados residentes en el Exterior, promovido por la Universidad de la República, el Ministerio de Relaciones Exteriores, la Organización 
Internacional para las Migraciones (OIM), el Programa de Naciones Unidas para el Desarrollo (PNUD) y el Fondo de Población de Naciones Unidas (UNFPA).

Ese año se crea por decreto la Comisión Nacional para la Vinculación con los Uruguayos Residentes en el Extranjero y un Comité Asesor de la Comisión, y comienza a sesionar una comisión parlamentaria para el estudio de respuestas legislativas a la problemática de la emigración. En 2002 se nombran los integrantes del Comité Asesor y comienzan a realizarse reuniones con migrantes, quienes proponen que el programa de vinculación no se reduzca a los "altamente calificados". El programa de vinculación se instrumenta entre 2001 y 2004 mediante un sitio web, una base de datos de 3000 registros voluntarios, un boletín informativo y la realización de talleres y reuniones anuales en Uruguay. En 2003, el comité asesor eleva a la Comisión Nacional un informe de evaluación y recomendaciones, que incluye más de cuarenta acciones orientadas a avanzar en la formulación de una política de estado en materia de vinculación con los uruguayos residentes en el exterior. Este documento resulta fundamental para comprender los antecedentes inmediatos de las acciones actuales en materia de vinculación con los uruguayos que residen en el exterior (Taks 2006). Los principales ejes de estas recomendaciones son: a) la creación de la Dirección General para la Comunidad de Uruguayos en el Exterior; b) el fortalecimiento del servicio consular; c) la promoción del intercambio científico y tecnológico; d) la promoción de la cultura y de la identidad nacional; e) la dinamización del intercambio económico y comercial; e) la generación de información sobre el tema migratorio, f) los apoyos a los compatriotas radicados en el exterior; g) el ejercicio de la ciudadanía por parte de la población emigrada; h) la revisión de la nacionalidad; y i) la promoción de instancias de intercambio.

Si bien, como se expuso brevemente, Uruguay da inicio a un proceso de revisión de su política migratoria-con especial énfasis en las estrategias de vinculación con los emigrantes - a partir del período de transición democrática, éste adquiere mayor intensidad durante el primer año de la primera gestión del Frente Amplio (2005), cuyo programa hace referencia al problema migratorio del Uruguay como nación, vinculado a los principales ejes del denominado "proyecto nacional de desarrollo productivo sustentable". Taks (2006) sostiene que la nueva política migratoria implementada por el gobierno frenteamplista 
puede analizarse en función a los siguientes ejes: a) la retención de población y el retorno de los emigrantes; b) la vinculación con los uruguayos que residen en el exterior; y c) la promoción de la inmigración de no-uruguayos. El Programa 2010-2015 del Frente Amplio confirma estos ejes prioritarios de la política migratoria uruguaya, afirmando que "El país todo debe reflexionar sobre esta emergencia [el fenómeno migratorio como problema demográfico en Uruguay], y para eso debe atender tres aspectos: la emigración/ migración/retención, la vinculación con los ciudadanos uruguayos en el exterior y la creación de condiciones para el retorno" ${ }^{\prime 15}$.

\section{Departamento 20 y Consejos Consultivos}

Si bien el programa de vinculación surge en 2001, como una apuesta del gobierno anterior y otras entidades y organismos, la primera administración del Frente Amplio reformula algunas prioridades del vínculo entre el Estado-nación y la población emigrada, con una apuesta al vínculo político y la participación ciudadana como principales objetivos de las acciones en esta materia. En 2005 se crea la Dirección de Asuntos Consulares y de Vinculación con los Uruguayos en el Exterior-denominada Departamento 20-en la órbita del Ministerio de Relaciones Exteriores. La denominación simbólica de Departamento 20—en relación a la división político-administrativa de Uruguay en 19 departamentos-señala el interés del gobierno en promover e institucionalizar canales oficiales de comunicación y vinculación entre el gobierno y los uruguayos que residen en el exterior. Esta propuesta se basa en tres elementos centrales: a) la creación de Consejos Consultivos en cada país donde haya una comunidad de uruguayos y representación del Servicio Exterior; b) la creación de un registro consular voluntario de emigrantes; y c) el aumento de la eficiencia del Servicio Exterior.

Algunas de las medidas llevadas a cabo por el Departamento 20 hasta la fecha han sido las siguientes ${ }^{16}$ : realización de "Operativos Celestes" (emisión de cédulas de identidad) en varias ciudades donde residen colectividades de uruguayos; implementación de mecanismos para el abaratamiento del envío de remesas; pago de jubilaciones y pensiones en el extranjero con la tarjeta VISA-BROU; lanzamiento de programa televisivo sobre la emigración uruguaya; experiencia piloto de cooperativas de vivienda para retornados; beneficios en telecomunicaciones; programa para uruguayos altamente calificados; acuerdo 
con la Intendencia de Montevideo para el envío gratuito de las partidas del Registro Civil; entre otras.

De acuerdo al sitio web del Departamento 20, los Consejos Consultivos constituyen organismos de la sociedad, que no responden ni están subordinados al Estado. Su principal objetivo es la unión de la colectividad uruguaya en los distintos países, en particular en aquellos en los que existe una representación del Servicio Exterior uruguayo. La única condición para la participación en los Consejos Consultivos es la ciudadanía uruguaya, siendo por tanto espacios abiertos a la pluralidad de posiciones políticas, religiosas, filosóficas, etc. El reconocimiento de los Consejos Consultivos como interlocutores legítimos del Estado uruguayo sólo requiere de dos condiciones: la inclusión de la totalidad de la colectividad uruguaya en el lugar que manifiesta su voluntad de participar y el respeto a una forma de funcionamiento democrático y transparente, que se exprese en elecciones periódicas de sus autoridades, funcionamiento abierto y cristalino, rendición de cuentas, etc. Cada Consejo Consultivo debe definir su estatuto de funcionamiento, su aprobación y puesta en práctica, en el entendido de que el relacionamiento con los nacionales que residen en el exterior debe ser flexible y respetar la auto-organización de las colectividades, con el objetivo de garantizar su protagonismo autónomo y activo. En este sentido, la participación en los Consejos Consultivos debe ser voluntaria, y sus estatutos de funcionamiento y agendas de actividades, autónomos y flexibles, únicamente limitados por las condicionantes ya mencionadas.

Los Consejos Consultivos no reemplazan al Servicio Exterior ni determinan su estructura de funcionamiento, sino que constituyen instancias ciudadanas para la manifestación de intereses, propuestas, demandas o sugerencias a las autoridades consulares. En lo que respecta a su agenda de prioridades y programa de realizaciones, los Consejos Consultivos son plenamente resolutivos y no deben rendir cuentas de sus acciones más que a sus integrantes. Las autoridades del Departamento 20 también manifiestan en su sitio web la intención de que, una vez que se reconozcan los derechos políticos que actualmente no se pueden ejercer desde el exterior del país, los Consejos Consultivos puedan contribuir a la promoción de la democracia. Una condición de los Consejos Consultivos es el Registro de Nacionalidad y Ciudadanía, a través del cual se pretende reafirmar la vinculación de los 
uruguayos del exterior, generando “(...) una formalidad con valor jurídico y vinculante que va mucho más allá que el interés por conocer el número y las características de la diáspora. Además de proporcionar información en tal sentido, a través del registro se persigue alentar esa reafirmación de la nacionalidad en la nueva ingeniería institucional que implica el Departamento 20"17. Las competencias de los Consejos Consultivos pueden ser muy amplias, comenzando con el mejoramiento del Servicio Exterior desde su papel de referentes de la sociedad civil en los distintos países. Además de este rol de control ciudadano, los Consejos Consultivos son espacios para la interacción entre miembros de una colectividad, con miras al intercambio cultural, la promoción del comercio y otras actividades económicas, la creación o mejoramiento de servicios, etc ${ }^{18}$.

De acuerdo a información del Departamento 20, a la fecha han sido creados más de 40 Consejos Consultivos, 20 de los cuales se encuentran en Argentina, Brasil, España y Estados Unidos. Hasta el momento se realizaron 3 encuentros de Consejos Consultivos (en 2006, 2007 y 2009), en los que participaron representantes de todo el mundo. El III Encuentro Mundial de Consejos Consultivos se realizó entre el 30 de marzo y el 4 de abril de 2009 en Montevideo. Participaron representantes de los Consejos Consultivos de Buenos Aires, Mar del Plata, Córdoba, Pelotas, Porto Alegre, Florianópolis, Asunción, Santiago de Chile, Puerto la Cruz, Valencia, Ciudad de México, Bajío mexicano, OrangeDover-Elizabeth-Manhattan, Québec, Cataluña, Tenerife, París, Gottemburgo, Sydney, Ilawarra y Melbourne. Algunos de los temas que se destacaron en la agenda del último encuentro fueron: la reglamentación de los Consejos Consultivos, la forma de comunicación entre la Dirección General para Asuntos Consulares y Vinculación y los Consejos Consultivos, la Oficina de Retorno y Bienvenida, la implementación de la "Tarjeta Celeste", los Operativos Cédula de Identidad, los convenios firmados por Uruguay con otros países en materia de previsión social, el recientemente instrumentado proyecto que facilita el envío de partidas de registro civil a los uruguayos que las requieran desde el exterior y la inclusión del Departamento 20 en los programas de estudio de la Enseñanza Primaria. La Declaración Final emitida por los Consejos Consultivos “(...) reafirma los valores fundacionales expresados desde el Primer Encuentro de la Patria Peregrina, sobre los cuales seguimos y queremos seguir construyendo nuestros espacios de participación ciudadana. Sentimos reafirmada nuestra autonomía y el reconocimiento como legítimos 
representantes de los uruguayos en el exterior." ${ }^{19}$ Por su parte, la Declaración Conjunta realizada por el Departamento 20 y los Consejos Consultivos presentes “(...) ratifica el compromiso con el proyecto del Departamento 20 y la aplicación de las políticas del Estado hacia los uruguayos residentes en el exterior y expresa que el desarrollo de este emprendimiento significa reafirmar el propósito en la ejecución de un camino de inclusión y amplificación de nuevos espacios para la práctica de la participación democrática y construcción de ciudadanía"20.

Sin embargo, el proceso de constitución de los Consejos Consultivos en los cuatro países en los que residen las principales comunidades de uruguayos (Argentina, Brasil, España y Estados Unidos $^{21}$ ) ha sufrido algunos obstáculos, que remiten, por un lado, a los rasgos específicos del asociacionismo anterior a esta iniciativa en cada uno de los países, y por otro, a las características sociodemográficas y los motivos de la emigración de los uruguayos residentes en esos países. En el caso de los uruguayos residentes en España, Moraes (2009) sostiene que el proceso de constitución de los Consejos Consultivos ha estado marcado por el temor a una institucionalización "desde arriba", ante lo cual se ha constituido un Comité por el Estatuto de los Ciudadanos Uruguayos en el Exterior, encargado de proponer mecanismos de coordinación entre las asociaciones y las formas organizativas propuestas por el gobierno uruguayo.

En el marco de este proceso de reconceptualización de la política migratoria uruguaya, en enero de 2008 se sanciona una nueva Ley de Migraciones (No 18.250) en Uruguay ${ }^{22}$. La formulación de esta ley busca adecuarse a los estándares internacionales del derecho internacional de los derechos humanos, aunque mantiene ciertas limitaciones marcadas por una concepción de seguridad en lo que respecta a las disposiciones sobre trata y tráfico de personas, frente a una realidad regional que avanza en materia de políticas de prevención de estos delitos (Taks 2010).

En materia de protección de los uruguayos que residen en el exterior, el artículo $71^{23}$ de la ley establece que el Estado uruguayo buscará suscribir convenios con los Estados en los que residen nacionales uruguayos, con miras a garantizarles la igualdad de trato con los nacionales de esos Estados. En la misma línea, el artículo $72^{24}$ dispone que el Poder Ejecutivo podrá suspender los beneficios que brinda la ley a los nacionales de los Estados 
que impongan restricciones a los uruguayos que se encuentren en su territorio con ánimo de permanencia, en atención al principio de reciprocidad.

En lo que respecta específicamente a la política nacional de vinculación y retorno de la emigración, el artículo $73^{25}$ de la ley dispone que la formulación de la misma esté a cargo del Ministerio de Relaciones Exteriores, a través del Departamento 20. Esta dependencia será la encargada de planificar, programar y ejecutar esta política a en el exterior a través del Servicio Exterior de la República, tomando en cuenta las sugerencias de los Consejos Consultivos.

El aspecto más relevante a tener en cuenta en el marco de este trabajo es que esta nueva normativa consolida a las iniciativas de vinculación con los uruguayos que residen fuera del país, y a los Consejos Consultivos en particular, como política de Estado. Así, el artículo 74 de la ley establece que "Los Consejos Consultivos son organizaciones representativas de los uruguayos residentes en el exterior cuyo cometido central será la vinculación con el país en sus más diversas manifestaciones. La organización y funcionamiento de los mismos se sustentará sobre la base de principios democráticos y la forma organizativa que establezca la reglamentación. El Servicio Exterior de la República, a través de sus misiones diplomáticas y oficinas consulares, los reconocerá como tales y brindará, dentro del ámbito de sus competencias, el apoyo que le sea requerido" (artículo 74 de la Ley No 18.250).

La ley también incluye disposiciones en materia de facilidades a aquellos ciudadanos uruguayos que deseen retornar al país tras haber residido en el exterior durante más de dos años (artículo 76 de la Ley 18.250) ${ }^{26}$ (Vaccotti 2011).

Si bien el Programa 2010-2015 del Frente Amplio reconoce que los Consejos Consultivos "todavía no han logrado concretar las dimensiones que se les dio en la propuesta inicial",27, insiste en la profundización de las estrategias de vinculación con los uruguayos que residen fuera del país, afirmando que "en un segundo período se elaborará un 'Plan nacional para los uruguayos residentes en el exterior' que continúe y profundice estas propuestas ya iniciadas, retomando su espíritu original, proyectándose en el tiempo un marco plural y transversal que involucre, a su vez a los países de acogida" 28 . 


\section{Conclusiones y reflexiones finales}

Como indican los datos brevemente expuestos en este trabajo, la emigración internacional no representa la misma problemática para Argentina y Uruguay. Aunque las grandes tendencias de la emigración internacional coinciden a grandes rasgos en ambos países, la importancia relativa de este fenómeno es mucho mayor en el caso uruguayo (frente a una importancia relativa mayor del fenómeno de la inmigración internacional en Argentina) representando así un problema demográfico de gran visibilidad y creciente presencia en la agenda política de Uruguay.

Si bien se observan diferencias significativas entre las estrategias llevadas a cabo por estos Estados para abordar el fenómeno de la emigración internacional, especialmente en términos de vinculación con las comunidades de emigrantes, es posible sostener que en ambos casos éstas no han sido sistemáticas ni integrales, al menos hasta el período reciente, en el que también se aprecian diferencias sustanciales entre ambos enfoques.

Aunque se registran iniciativas aisladas-a partir de la década de 1960 en Argentina y de 1980 en Uruguay_es sólo en el período reciente que se observa un conjunto de acciones coordinadas orientadas a reformular el vínculo entre estos Estados y sus comunidades de emigrantes, en ambos casos con una clara apuesta a la participación ciudadana de los emigrantes y a la democracia representativa, aunque con diferentes matices y énfasis.

La principal medida adoptada específicamente en materia de vinculación con la emigración internacional es la creación del programa Provincia 25 en Argentina y del Departamento 20 y los Consejos Consultivos en Uruguay.

Sin embargo, ambas iniciativas presentan diferencias. En lo que respecta a la consolidación normativa de ambas iniciativas, se observa que mientras que en Argentina, la formulación de una ley migratoria que contempla medidas concretas para el abordaje de la vinculación con la emigración (2004) precede a la creación del programa Provincia 25 (2007) y, por ende, no lo incluye, en Uruguay el proceso se da a la inversa: el Departamento 20 y los Consejos Consultivos surgen en 2005 y son posteriormente consolidados mediante su inclusión en la ley migratoria sancionada en 2008, fuertemente inspirada en la normativa argentina, que es considerada pionera en muchos aspectos, principalmente en lo que respecta al tratamiento de la inmigración internacional. 
En relación a esto último, se observan dos grandes similitudes en lo que respecta a las disposiciones en materia de tratamiento de la emigración internacional incluidas en ambas leyes: el fomento a la suscripción de convenios con países donde residan nacionales con miras a garantizarles mejores condiciones de vida, y las facilidades para aquellos nacionales que decidan retornar al país.

Es fundamental destacar que ambos marcos normativos para la gobernabilidad de la migración merecen un análisis en mayor profundidad, especialmente desde una perspectiva de derechos, que incluya, por ejemplo, un seguimiento de los contenidos de los convenios firmados con otros países, que incluyan disposiciones en materia de protección de los derechos de los emigrantes.

En el caso de Argentina, la Ley 25.871 de 2004 ha recibido gran atención, en virtud de los importantes avances que representa, especialmente en lo que refiere al tratamiento de la inmigración internacional, tema que ostenta — y que ha ostentado históricamente — un lugar significativamente más relevante que la emigración internacional en la agenda política del país.

Las transformaciones brevemente expuestas en la política de vinculación de ambos Estados se enmarcan en tendencias que datan de la década de 1990, aunque su presencia en la agenda internacional sólo se observa a partir de la década de 2000. Las estrategias de vinculación de los Estados con las comunidades de emigrantes han sido abordadas fundamentalmente desde la perspectiva transnacional, aunque los estudios sobre los casos argentino y uruguayo aún son escasos.

En este sentido, un primer elemento a analizar tiene que ver con la participación de los emigrantes en el proceso de definición y ejecución de la política de vinculación. Mientras que en Argentina no se observa una fuerte participación de los migrantes en la formulación e implementación de la política de vinculación, en Uruguay sí se registra este tipo de participación, incluso desde iniciativas anteriores a 2005, como, por ejemplo, en el Comité Asesor de la Comisión Nacional para la Vinculación con los Uruguayos Residentes en el Extranjero (2001). No obstante, en ambos casos se carece de estudios que aborden estos procesos de definición e implementación de políticas de vinculación que privilegien una mirada a las formas de participación de los migrantes en las mismas. 
Más allá de esta precisión, es posible sostener que a partir del surgimiento de estas iniciativas se amplía el marco formal para la participación de los emigrantes en la definición de las prioridades de la política de vinculación de ambos Estados, aunque más explícitamente en el caso uruguayo, con la creación y posterior consolidación normativa de los Consejos Consultivos. Sin embargo, por el momento no existen estudios que analicen el funcionamiento de estos programas desde su creación hasta la fecha, ni la posibilidad de considerarlos como espacios transnacionales consolidados. En relación a esto último, sería interesante analizar cuáles han sido las acciones y propuestas surgidas de estos espacios en relación a ambos estados, de qué forma pueden clasificarse, y cómo se vinculan con las características sociodemográficas de los participantes y los motivos y expectativas de dicha participación.

En esta misma línea, y como ya fue mencionado anteriormente, las relaciones transnacionales surgen del interés de los migrantes de sobreponerse a diversas situaciones de subordinación en los países de origen y destino (Portes 1999), por lo que resulta relevante indagar acerca de las trayectorias de participación/asociación-tanto en Argentina y Uruguay como en los países de destino-de las personas que participan en estos espacios. En esta línea, sería interesante preguntarnos por qué estás personas eligen participar en estos espacios, ya sea como alternativa a espacios de participación ciudadana más tradicionales de la emigración de estos países, o en simultáneo a los mismos, y cuáles son sus motivaciones y expectativas específicas con respecto a estos espacios.

Otro aspecto relevante tiene que ver con el marco institucional en el que se insertan las iniciativas de vinculación. Mientras que el Estado argentino crea un programa en el marco del Ministerio del Interior, el uruguayo crea una división administrativa en el Ministerio de Relaciones Exteriores. Es necesario contar con estudios que analicen comparativamente las posibilidades de estas nuevas ingenierías institucionales de contribuir a la formación de colectivos transnacionales desterritorializados y vinculados de diversas formas a Argentina y Uruguay.

Otro tema que demanda análisis en mayor profundidad es el de los procesos de extensión de derechos políticos a los emigrantes en el marco de las políticas de vinculación de estos estados. En Argentina el derecho al voto desde el exterior se encuentra consagrado desde 
1991, aunque con niveles de inscripción bajos y decrecientes. Asimismo, existe un proyecto de ley para la creación de un distrito exterior, que habilite la representación parlamentaria de los argentinos residentes en el exterior, aunque por el momento no hay avances al respecto. Ambos temas requieren de un atento seguimiento, en virtud del potencial que implican.

Por su parte, el derecho al voto desde el exterior aún no se encuentra reconocido en Uruguay, más allá de representar una de las principales demandas de las colectividades de uruguayos en el exterior desde la salida de la dictadura. En 2009 se plebiscitó esta opción, junto con las elecciones nacionales, pero no logró la aprobación, alcanzando sólo un 37\% de los votos. Este resultado constituyó un fuerte revés a la política de vinculación, generando expectativas y necesidad de seguimiento de las estrategias que adopte la segunda administración del Frente Amplio (2010-2015) para dar cumplimiento a esta demanda. En lo que respecta a la posibilidad de representación parlamentaria de los uruguayos que residen en el exterior, por el momento no se han presentado proyectos de ley al respecto.

En materia de vinculación en el campo económico, las políticas llevadas a cabo por ambos estados incluyen una serie de acciones, aunque en ambos casos de carácter aún incipiente. Estas medidas también demandan análisis más profundos, que presten especial atención a las especificidades de la emigración de estos dos países, por ejemplo, la menor importancia relativa de las remesas en comparación con otros países.

En lo que respecta a la vinculación en el plano cultural, en el caso uruguayo se observa un número de acciones de carácter simbólico, orientadas a promover una reafirmación "identitaria"-como el lanzamiento de programas televisivos y radiales sobre la emigración uruguaya, el otorgamiento de beneficios en telecomunicaciones y el apoyo institucional a la organización de eventos vinculados a las tradiciones y valores uruguayos en el exterior (Taks 2010)—que también merecen especial atención, en relación a su potencial de contribuir a la consolidación de espacios sociales transnacionales.

Asimismo, son varias las preguntas de investigación que surgen desde la perspectiva demográfica. Resulta interesante preguntarnos quiénes participan de los espacios recientemente creados por ambos estados, cuáles son las características sociodemográficas de estas personas (edad, sexo, nivel educativo, composición del núcleo familiar, ocupación, 
ingreso, etc), y si puede observarse alguna correspondencia con el perfil sociodemográfico estimado de la emigración de ambos países en general y de cada país en particular.

En síntesis, si bien se resaltan los aspectos positivos de las nuevas políticas de vinculación de Argentina y Uruguay, su carácter reciente, sus consiguientes limitaciones y los numerosos interrogantes que plantean señalan la necesidad de esfuerzos académicos orientados a conocer más acerca de su proceso de formulación e implementación, así como sobre sus impactos, con el objetivo de contribuir a su desarrollo.

\section{Referencias}

Aguiar, César (2008) "Inmigración. Perspectivas y oportunidades para una política inmigratoria". En Juan José Calvo y Pablo Mieres (eds) Sur, migración y después. Propuestas concretas de políticas de población en Uruguay. Montevideo: UNFPA y Rumbos, 2008.

Bertoncello, Rodolfo y Lattes, Alfredo (1986) “Medición de la Emigración de Argentinos.a partir de la información nacional". En Oteiza, Enrique y Lattes Alfredo Dinámica migratoria argentina (1955-1984): Democratización y retorno de expatriados. Ginebra: UNRISD-CENEP, 1986: 34-65.

Bengochea, Julieta y Cabrera, Mariana (2010) "Propensión migratoria de los jóvenes: Uruguay en perspectiva comparada 1990-2008”, IV Congreso de la Asociación Latinoamericana de Población, La Habana, Cuba, 16 al 19 de noviembre de 2010.

Borraz, Fernando y Pozo, Susana (2007) "Remittances in Uruguay". Revista de Ciencias Empresariales y Economía 1: 19-45.

Cabella, Wanda y Pellegrino, Adela (2007) "Emigración. Diagnóstico y aportes para discutir políticas". En Juan Calvo y Pablo Mieres Importante pero urgente. Políticas de población en Uruguay. Montevideo: UNFPA y Rumbos, 2007. 
(2005) "Estimación de las migración internacional en Uruguay 1963-2000". Serie documentos de trabajo $\mathbf{N}^{\mathbf{0}}$ 70. Montevideo: Unidad Multidisciplinaria /Facultad de Ciencias Sociales.

Canales, Alejandro y Zlolniski, Christian (2001) "Comunidades transnacionales y migración en la era de la globalización”. Notas de Población 73.

Calvelo, Laura (2007) "La emigración en Argentina: 1960-2000". En Susana Torrado (comp) Población y bienestar en la Argentina del primero al segundo Centenario. Tomo I. Buenos Aires: Editorial Edhasa, 2007: 212-236.

CEPAL (2006 ${ }^{a}$ Cuatro temas centrales en torno a la migración internacional, derechos humanos y desarrollo (LC/L.2490), Santiago de Chile.

(2006b) Migración internacional, derechos humanos y desarrollo en América Latina y el Caribe, Síntesis y Conclusiones (LC/G.2303(SES.31/11), Santiago de Chile.

--------: (2002) Globalización y desarrollo, (LC/G.2157(SES.29/3)), Santiago de Chile.

Diconca, Beatriz y De Souza, Lydia (2003) "El camino del 'afuera'. La opción de emigrar". En Anuario de Antropología Social y Cultural en Uruguay 2002-2003, Montevideo.

Escriva, Ángeles (2009) "Formas y motivos de la acción trasnacional. Vinculaciones de los peruanos con el país de origen". En Migración y participación política. Estados, organizaciones y migrantes en perspectiva local-transnacional, Córdoba: CSIC.

Faist, Thomas (2005) "Espacio social transnacional y desarrollo: una exploración de la relación entre comunidad, Estado y mercado". Revista Migración y Desarrollo, 5, segundo semestre.

Falero, Alfredo (2002) "Migración laboral: un desafío para la sociedad civil". Revista de Ciencias Sociales, Departamento de Sociología, Año XV, №20. 
Frente Amplio (2008) Programa 2010 -2015. Aprobado por el V Congreso Extraordinario "Zelmar Michelini 13 y 14 de diciembre de 2008. http://www.pvp.org.uy accesado el 1 de enero de 2011.

Glick Schiller, Nina y Fouron, George (2011) George woke up laughing: long-distance nationalism and the search for home. Durham: Duke University Press.

Glick Schiller, Nina; Basch, Linda y Szanton-Blanc, Cristina (1999) "From immigrant to transmigrant: theorizing transnational migration". En Ludger Pries (ed.) Migration and transnational social spaces. Ashgate: Aldershot.

Guarnizo, Luis Eduardo (2004) "Aspectos económicos del vivir transnacional”. En Escriva, Ángeles y Ribas, Natalia (coords.) Migración y desarrollo. Estudios sobre remesas y otras prácticas transnacionales en España, Córdoba: CSIC.

Guarnizo, Luis Eduardo; Portes, Alejandro y Haller, William (2003) "Assimilation and transnationalism: determinants of transnational political action among contemporary migrants”. American Journal of Sociology, Vol. 108, º 6.

Gurrieri, Jorge (1982) Emigración de Argentinos. Una estimación de sus volúmenes. Buenos Aires: Dirección Nacional de Migraciones.

Levitt, Peggy y Nyberg-Sorensen, Ninna (2004) "The transnational turn in migration studies”, Global Migration Perspectives, $\mathrm{N}^{\circ} 6$.

Levitt, Peggy y Glick Schiller, Nina (2004) "Perspectivas internacionales sobre migración: conceptualizar la simultaneidad" Revista Migración y Desarrollo, $\mathrm{N}^{\circ}$ 3, segundo semestre.

Macadar, Daniel, y Pellegrino, Adela (2007) Informe de migración internacional. Encuesta Nacional de Hogares Ampliada. Montevideo, Montevideo: Instituto Nacional de Estadística (INE). 
Martínez Pizarro, Jorge (2001) Reflexiones sobre la gobernabilidad de la migración internacional en América Latina. Revista Migraciones Internacionales, Vol. 1, Núm. 1, julio-diciembre.

Mato, Daniel (coord.) (2003) Políticas de identidades y diferencias sociales en tiempos de globalización. Caracas: Universidad Central de Venezuela.

(2004) Políticas de ciudadanía y sociedad civil en tiempos de globalización. Caracas: Universidad Central de Venezuela.

Moraes, Natalia (2007) "Identidad transnacional, diáspora/s y nación: Una reflexión a partir del estudio de la migración uruguaya en España”. En Mato, Daniel y Maldonado, Fermín: Cultura y Transformaciones sociales en tiempos de globalización. Perspectivas latinoamericanas.

Moraes, Natalia; Bermudez, Anastasia; Escriva, Ángeles y Padilla, Beatriz (2009): "Estrategias de vinculación de los estados latinoamericanos con sus diásporas: Un análisis de las iniciativas desarrolladas por Colombia, Perú, Brasil y Uruguay". Migración y participación política. Estados, organizaciones y migrantes en perspectiva localtransnacional, Córdoba: CSIC.

Moreira, Constanza y Pellegrino, Adela (2001) "Ciudadanía y migración: las fronteras del Uruguay como comunidad política”. En Laura Gioscia (comp.) Ciudadanía en tránsito. Montevideo: Ediciones de la Banda Oriental.

Notaro, Jorge; Canzani, Agustín; Longhi, Augusto y Méndez, Estela (1990) "El retorno y las respuestas de la sociedad uruguaya". En La migración de retorno. Montevideo: CIEDUR/FCU.

Novick, Susana (Dir).2007 Sur-Norte. Estudios sobre la emigración reciente de argentinos. Buenos Aires: Catalogos-UBA.

OIM (2008) Perfil migratorio de Argentina 2008, Buenos Aires: OIM. 
Ostergaard-Nielsen, Eva (ed) (2003) International Migration and Sending Countries: Perceptions, Policies and Transnational Relations, Londres: Palgrave Macmillan Ltda.

Pazos, Nora (2009) "Emigración y polìticas de vinculación en Argentina y Uruguay". VII Encontro Internacional Fórum Universitário MERCOSUL, Rio de Janeiro, noviembre.

Pedone, Claudia (2002) "El potencial del análisis de las cadenas y redes migratorias en las migraciones internacionales contemporáneas". III Congreso sobre la Inmigración, España, Granada, noviembre.

Pellegrino, Adela (2009) Uruguay. País de migrantes internos y externos Montevideo: PNUD. (2008) "Las políticas de migración y desarrollo". En Calvo, Juan José y Mieres, Pablo (Editores) Sur, migración y después. Propuestas concretas de políticas de población en Uruguay. Montevideo: UNFPA y Rumbos.

-(2006) "La migración calificada en América Latina", Encuentro Iberoamericano sobre Migración y Desarrollo, Secretaría General Iberoamericana, Madrid. Uruguay, OIM.

-(2003 $)$ Migración de mano de obra calificada desde Argentina y

(2003b) Caracterización demográfica del Uruguay Montevideo: UDELAR/UNFPA.

(2003c) "Uruguay y la ciudadanía dispersa”. En Calderón Chelius (comp) Votar en la distancia. La extensión de los derechos políticos a migrantes, experiencias comparadas. México, Instituto Mora.

-(1995) Migración e integración. Nuevas formas de movilidad de la población Montevideo: Facultad de Ciencias Sociales (Universidad de la República) y Trilce. 
Pellegrino, Adela y Vigorito, Andrea (2003) Emigration and economic crisis: recent evidence from Uruguay Montevideo.

Pellegrino, Adela; Vigorito, Andrea y Macadar, Daniel (2003) Informe sobre emigración y remesas en Uruguay, Banco Interamericano de Desarrollo.

Pellegrino, Adela y Martínez Pizarro, Jorge (2001) "Una aproximación al diseño de políticas sobre la migración internacional calificada en América Latina". Población y Desarrollo, $\mathrm{N}^{\circ} 23$, diciembre.

Pellegrino, Adela y Luján, C. (1994) La propensión migratoria de los jóvenes uruguayos. Estudio en base a datos de la Encuesta Nacional de Juventud 1989-1990 del Instituto Nacional de Estadística, Montevideo: INJU, CEPAL y OIM.

Portes, Alejandro (2005) “Convergencias teóricas y evidencias empíricas en el estudio del transnacionalismo de los inmigrantes”. Revista Migración y Desarrollo, primer semestre.

-(1999) "Conclusion: towards a new world - the origins and effects of transnational activities”. Ethnic and Racial Studies, vol. 22, N² 2, marzo de 1999.

Portes, Alejandro; Guarnizo, Luis E. y Haller, William (2002) "Transnational entrepreneurs: an alternative form of immigrant adaptation". American Sociological Review, vol 67, $\mathrm{N}^{\circ}$ 2, abril.

Portillo, Álvaro (2006) "La política migratoria del Estado uruguayo", Encuentro Iberoamericano sobre Migración y Desarrollo, 18 y 19 de julio de 2006.

Portillo, Álvaro (1989) Migración interna e internacional en el Uruguay. Montevideo: Fundación de Cultura Universitaria.

Supervielle, Marcos (1989) "Recuento histórico de las políticas migratorias en el país y propuestas de nuevas políticas". Cuadernos de la Facultad de Derecho y Ciencias Sociales, Volumen II, número 11: pp. 115-136. 
Taks, Javier (2010): “Antecedentes y desafíos de las políticas de migración en Uruguay”. En Zurbriggen, Cristina y Mondol, Lenin Estado actual y perspectivas de las políticas migratorias en el MERCOSUR, Montevideo: FLACSO Uruguay/UNESCO.

(2006) "Migraciones internacionales en Uruguay: de pueblo trasplantado a diáspora vinculada" Revista Theomai 14.

Trigo, Abril (2003) Memorias migrantes: testimonios y ensayos sobre la diáspora uruguaya, Montevideo: Beatriz Viterbo/Trilce.

Vaccotti, Luciana (2011) "Transnacionalismo, emigración internacional y políticas de vinculación en Uruguay". En Martínez Pizarro, Jorge (Editor) Colección de ensayos sobre población y derechos humanos en América Latina, Río de Janeiro: ALAP, 2011: 195220.

Varela Petito, Carmen (Coord.) (2008) Demografía de una sociedad en transición. La población uruguaya a inicios del siglo XXI, Montevideo: Trilce.

Vono De Vilhena, Daniela (2006) “Vinculación de los emigrados latinoamericanos y caribeños con su país de origen: transnacionalismo y políticas públicas” Revista Población y Desarrollo 71.

$1 \quad$ Decreto $7558 / 65$

2 Reglamentada por el Decreto $1138 / 93$ y modificada por el Decreto 2010/93 y Decreto Nacional $254 / 09$.

3 "Los ciudadanos argentinos que, residiendo en forma efectiva y permanente fuera del territorio de la República Argentina, sean electores nacionales de acuerdo a lo dispuesto en el Código Electoral Nacional y se inscriban en el Registro de Electores Residentes en el Exterior establecido en el artículo siguiente, podrán votar en las elecciones nacionales" (artículo 1 de la Ley 24.007)

$4 \quad$ Fundamentación del Decreto 452/2007

5 www.cancilleria.gov.ar/portal/Elecciones2011, accesado el 20 de agosto 2011

6 Reglamentada por el Decreto 616/2010 
7 "El gobierno de la República Argentina podrá suscribir convenios con los Estados en los que residan emigrantes argentinos para asegurarles la igualdad o asimilación de los derechos laborales y de seguridad social que rijan en el país receptor. Dichos tratados deberán asimismo garantizar a los emigrantes la posibilidad de efectuar remesas de fondos para el sostenimiento de sus familiares en la República Argentina. El Poder Ejecutivo podrá suspender los beneficios otorgados por la presente ley respecto de los súbditos de aquellos países que tengan establecidas restricciones para los ciudadanos argentinos allí residentes, que afecten gravemente el principio de reciprocidad.” (artículo 102 de la Ley 25.871)

8 "Todo argentino con más de dos (2) años de residencia en el exterior que decida retornar al país podrá introducir los bienes de su pertenencia destinados a su actividad laboral libre de derechos de importación, tasas, contribuciones y demás gravámenes, así como su automóvil, efectos personales y del hogar hasta el monto que determine la autoridad competente, hasta el monto y con los alcances que establezca el Poder Ejecutivo nacional” (artículo 103 de la Ley 25.871)

9 “Las embajadas y consulados de la República Argentina deberán contar con los servicios necesarios para mantener informados a los argentinos en el exterior de las franquicias y demás exenciones para retornar al país" (artículo 104 de la Ley 25.871)

$10 \quad$ Resolución 452 de 2007

11

http://www.mininterior.gov.ar/provincias/p25_mision.php?idName=provincias\&idNameSubMenu=p rovinciasProv25\&idNameSubMenuDer=intProvProv25Mision, accesado el 20 de agosto 2011.

12 http://www.bapro.com.ar/banco/ed20081125-SIUW.htm, accesado el 20 de agosto 2011.

13 http://www.mininterior.gov.ar/provincias/p25 guia.php

$14 \quad$ Expediente 3841-D-2009

15 Programa 2010 - 2015 del Frente Amplio, aprobado en el V Congreso Extraordinario "Zelmar Michelini” del Frente Amplio, 13 y 14 de diciembre de 2008:160.

16 Elaborado en base a boletines informativos publicados en http://www.d20.org.uy, accesado el $10 / 05 / 2011$

17 http://www.mrree.gub.uy/gxpsites/hgxpp001?7,3,189,O,S,0,MNU;E;13;5;MNU; accesado el $10 / 05 / 2011$

18 http://www.mrree.gub.uy/gxpsites/hgxpp001?7,3,189,O,S,0,MNU;E;13;5;MNU; $\quad$ accesado el $10 / 05 / 2011$. 

accesado el 20/11/2009.

20 Boletín Informativo del Departamento 20 de junio de 2009, publicado en http://www.d20.org.uy, accesado el 20/11/2009.

21 El sitio web del Departamento 20 registra 50 organizaciones de uruguayos en España, 6 en Brasil y 8 en Estados Unidos, no existiendo información sistematizada para el caso de Argentina (http://www.mrree.gub.uy/gxpsites/hgxpp001?7,3,294,O,S,0,MNU;E;11;4;MNU;, accesada el 10/05/2011.

$22 \quad$ Esta ley derogó la Ley 8.868 de 1932. Se reglamenta en 2009.

23 "El Estado uruguayo fomentará la suscripción de convenios con los Estados en los que residen nacionales uruguayos, a los efectos de garantizarles la igualdad de trato con los nacionales de esos Estados" (artículo 71 de la Ley 18.250)

${ }_{24} \quad$ "El Poder Ejecutivo podrá suspender los beneficios que otorga la presente ley a los nacionales de los Estados que dicten normas o reglamentos que dispongan restricciones a los uruguayos que se encuentren en el territorio de dichos Estados con ánimo de permanencia, en tanto se afecte el principio de reciprocidad" (artículo 72 de la Ley 18.250)

25 "El Ministerio de Relaciones Exteriores, a través de la Dirección General para Asuntos Consulares y Vinculación, tendrá a su cargo la coordinación de la política nacional de vinculación y retorno con la emigración. Planificará, programará y ejecutará dicha política en el exterior a través del Servicio Exterior de la República, el que considerará especialmente las sugerencias que al efecto emitan los Consejos Consultivos en cuanto fuera pertinente" (artículo 73 de la Ley 18.250)

26 "Todo uruguayo con más de dos años de residencia en el exterior que decida retornar al país, podrá introducir libre de todo trámite cambiario y exento de toda clase de derechos de aduana, tributos o gravámenes conexos: A) Los bienes muebles y efectos que alhajan su casa habitación; B) Las herramientas, máquinas, aparatos e instrumentos vinculados con el ejercicio de su profesión, arte u oficio; C) Por una única vez, un vehículo automotor de su propiedad, el que no podrá ser transferido hasta transcurrido un plazo de cuatro años a contar desde su ingreso a la República. El régimen a que esté sujeto el automotor deberá constar en los documentos de empadronamiento municipal y en el Registro Nacional de Automotores. El citado vehículo deberá ser empadronado directamente por la persona interesada en la Intendencia Municipal correspondiente" (artículo 76 de la Ley 18.250).

27 Programa 2010 - 2015 del Frente Amplio, aprobado en el V Congreso Extraordinario "Zelmar

Michelini”, 13 y 14 de diciembre de 2008: 161.

28 Programa 2010 - 2015 del Frente Amplio, aprobado en el V Congreso Extraordinario "Zelmar

Michelini”, 13 y 14 de diciembre de 2008: 161. 